\title{
El problema de la interdisciplina en la comunicación organizacional. Algunos avatares epistemológicos.
}

Rafael Ávila González-UAM Cuajimalpa- ravila99@gmail.com José Alfredo Andrade García-UAM Cuajimalpa- afredo@hotmail.com

\section{Resumen:}

La cuestión de la usabilidad de la comunicación organizacional ha dominado históricamente este campo de estudios. Por lo mismo, consideramos que esa matriz pragmática tiene necesidad de sustento teórico y epistemológico que trascienda el determinismo tecnoeconómico y lo justifiquen como dominio de acción colectiva. Pensamos que una de las posibles vías de renovación de este ámbito radica en la posibilidad de explicitar cuestiones formales, técnicas y, de hecho, filosóficas, en cuanto a sus motivaciones y estándares de actuación. Para ello precisamos escrutar las representaciones que, en calidad de imperativos categóricos, modelan los ámbitos organizacionales en diferentes planos y distintas modalidades, originadas a su vez en diversas disciplinas epistémicas. Nos proponemos explorar algunos lugares de origen de tales representaciones y la función asignada a la comunicación, más allá del dispositivo sistémico, en tanto práctica social desontologizada. En tal virtud, la comunicación organizacional es cuestionada en su discurso monodisciplinar y se hace necesaria una justificación inter o transdisciplinar para dotarla de credibilidad y validez. Poner de relieve esa estructura gnómica en esta materia es el objetivo del presente trabajo, que continúa además otros esfuerzos orientados en esa dirección. 


\section{Las nuevas narrativas, en el entorno social Universidad de La Laguna, diciembre de 2019}

Palabras clave: Organización, comunicación organizacional, trabajo, interdisciplina, transdisciplina.

\section{Introducción.}

Este trabajo bien podría llevar por título "Segunda parte de 'organización, comunicación y cultura: opciones supradisciplinares'”, en virtud de que arecientemente se publicó un artículo con ese nombre en la revista digital Organicom (Ávila y Andrade, año 16, número 30, 2019: 65-81). Entre ambos forman unidad, y si no fue posible ofrecerlos juntos se debió sobre todo a motivos técnicos. La idea regulativa es inquirir por lo que tiene de distintivo el fenómeno organizacional en tanto que "hecho organizado", es decir, en cuanto factum operacional de la razón con determinadas características. Pensamos que ese es el camino adecuado para poder preguntar por la naturaleza de la cultura y la comunicación organizacionales.

Tal como se concibe en las literaturas correspondientes, la "organización" es el horizonte de acción, la cultura su expresión identitaria-diferenciadora, fundamentalmente de orden simbólico, y la comunicación su energeia, su acto realizador al proveer de un fondo de sentido las interacciones y mandatos operacionales y estratégicos

Se comprende, entonces, que la intención de fondo es preguntar acerca de las posibilidades de acceso al conocimiento de la cultura y la comunicación organizacionales tomando como punto de partida las determinaciones epistémicas de la forma "organización", considerando hipotéticamente que el modo como se concibe la naturaleza organizacional determina en primera instancia la manera en que se tratan, "en la realidad", tanto la comunicación como la cultura. La pregunta central es sobre la posibilidad de evadir tales determinaciones. 


\section{Las nuevas narrativas, en el entorno social Universidad de La Laguna, diciembre de 2019}

En este sentido hay una diferencia sustancial entre ambos textos: mientras en aquél decidimos seguir un orden analítico para dar razón de la organización del conocimiento, en este las tematizaciones "surgen" de la propia reflexión al intentar precisar las vías de abordaje de esa complejidad que en tanto nexo forman los tres "campos" de conocimiento susodichos.

\section{De las "opciones supradisciplinares" a los avatares epistemológicos.}

Para dar forma a ese propósito ha sido necesario trabajar en dos direcciones: a) en la constitución histórica de las denominaciones disciplinares, entendiéndolas como la forma institucional de organizar el conocimiento, e incorporando el momento actual como el de un cuestionamiento de la monodisciplina y la transición hacia formas supradisciplinares, lo que significaría una reorganización legaliforme del saber; b) en el análisis de los conceptos concurrentes en el estudio de la Comunicación organizacional, revisando el modo como se constituye la organización en tanto que organización, su conceptualización y las determinaciones cognitivas que orientan su operación.

De modo que aquí volveremos al problema de la supradisciplina pero con un nuevo punto de partida. Si allá optamos por la configuración disciplinar y sus implicaciones institucionales en el plano histórico occidental, aquí nos concentramos en lo relativo a la "organización" como sujeto de interrogación. Pensamos que de este modo es posible relativizar las formulaciones disciplinares que subordinan los fenómenos cultural y comunicacional a la racionalidad teleológica del agente organizacional, al tiempo que se postula una nueva apertura del "mundo" de las organizaciones a intereses legítimamente diversos.

Con el cuadro que reinstalamos en la siguiente página intentamos dar sentido amplio a tales opciones que llamamos supradisciplinares. Hemos añadido los títulos 


\section{Las nuevas narrativas, en el entorno social Universidad de La Laguna, diciembre de 2019}

de la izquierda con el propósito de unificar la interpretación, pero básicamente es la propuesta de J. Thompson.

Atendiendo sobre todo las flechas de cada ítem, vemos que el enfoque epistémico es tan amplio que va desde la complementación, propia de la multidisciplina, hasta la franca hibridación en la opción transdiciplinar; el interés metodológico, por su parte, contempla un continuum integrador, mientras que en la investigación y desarrollo (I\&D) los extremos son la cooperación entre una y otra (investigación y desarrollo, se entiende), y la co-ordinación de la función desarrolladora en función del interés unificador y la integración epistémica.

¿Qué significa eso para los efectos de lo que llamamos "avatares epistemológicos"? En primer lugar, que la cuestión de las definiciones conceptuales, es decir, teóricas, Tabla 1. Las formas tipo en cuanto a Investigación y desarrollo.

\begin{tabular}{|c|c|c|}
\hline Multidisciplinariedad & Interdisciplinariedad & Transdisciplinariedad \\
\hline \multicolumn{3}{|l|}{ Enfoque epistémico } \\
\hline Yuxtaposición & Complementación & Disrupción / Transgresión \\
\hline Secuenciación & Interacción & Transformación \\
\hline \multirow[t]{2}{*}{ Concurrencia } & Eslabonamiento & Transposición \\
\hline & Re-focalización & Innovación \\
\hline Complementación & $4 \ldots \ldots$ & Hibridación \\
\hline \multicolumn{3}{|l|}{ Interés metodológico } \\
\hline Indiscriminado & & Transgenérica (campos) \\
\hline Enciclopédico & & Integración epistemológica \\
\hline Integración parcial & $\longrightarrow$ & Integración total \\
\hline \multicolumn{3}{|l|}{ Tendencias de I\&D } \\
\hline Coyuntural & & Estratégica \\
\hline Auxilio I->D & Complementariedad & Estructural \\
\hline Suplementarias D\&I & Generalización (abstracta) & Unificadora (práctica) \\
\hline
\end{tabular}




\section{Las nuevas narrativas, en el entorno social}

Universidad de La Laguna, diciembre de 2019

Grados de colaboración

\begin{tabular}{ll}
\multicolumn{1}{c}{ Grados de colaboración } \\
I\&D co-operativa & \\
\hline I\&D Reducción binaria & Estrecha vs amplia \\
Metodológica vs teórica \\
Puenteo vs reestructuración \\
Instrumental vs crítica \\
Endógena vs exógena
\end{tabular}

Fuente: Tomado de Thompson, J., A taxonomy of interdisciplinarity.

resiente una pérdida de importancia frente al interés primordialmente técnico, comprendido como razón instrumental, de la ciencia y sus métodos presumiblemente duros.

En segundo lugar, ese rezago del trabajo metodológico (de naturaleza teórica, otra vez), expresa a su vez la vulnerabilidad de la reflexión epistemológica frente a las exigencias tecnológicas de las aplicaciones científicas.

Las determinaciones exógenas del aparato tecnocientífico, mismas que se verifican en la organización del conocimiento efectuado a partir de las monodisciplinas, hacen necesario, pensando ante todo en nuestros objetos, abrir la discusión en clave fenomenológica, tal que los distintos saberes que concurren en su estudio (teorías de las organizaciones, ciencias de la gestión, simbolismo organizacional, cultura organizacional, comunicación organizacional...) puedan conectar en conversaciones productivas manteniendo a salvo los órdenes disyuntos de pensamiento que recíprocamente representan, pero sin menoscabo de las oportunidades que se presenten para poder converger en estudios comprehensivos sobre el terreno. 


\section{Las nuevas narrativas, en el entorno social Universidad de La Laguna, diciembre de 2019}

Dicho de otro modo: el fin de la supradisciplina (refiriéndonos en realidad tan solo a la inter y la transdisciplina) no puede ser, como pensaba Nicolescu, la constitución de nuevas fronteras disciplinares, así sea emergentes pero definibles a título de campos permeables y de contornos problemáticos. Tampoco puede serlo, como proponían el empirismo lógico o la filosofía analítica, propiciar la unidad de la ciencia (de toda ciencia en una plenamente arbitrada) bajo criterios de cientificidad uniformes, toda vez que los temas, intereses, miradas, abordajes, compuestos y yuxtaposiciones que implica la conformación de los objetos, son de tal forma disímbolos que una unificación formal parecería más un schema de ordenación aristotélico, que una coexistencia intercomprensiva de lo diverso.

Es indudable que los esfuerzos por penetrar las fronteras disciplinares han dado suficiente materia para alimentar las discusiones académicas, sin embargo, la realidad es que las instituciones nacionales e internacionales que gestionan la organización del conocimiento, es decir, que apoyan financieramente su producción y evalúan sus resultados, siguen anclados en la base disciplinar para realizar su trabajo. Así lo dejan entrever, por ejemplo, los informes del Conacyt en México, tanto en la oferta educativa como en la investigación y desarrollo.

La tabla fue elaborada con los informes oficiales y las negrillas indican en dónde hubo cambios de un reporte al otro.

Tabla 2. Organización del conocimiento por el Conacyt.

\begin{tabular}{|c|c|c|}
\hline $\begin{array}{l}\text { Año de } \\
\text { informe }\end{array}$ & "Áreas" en educación superior (Tercer nivel) & $\begin{array}{l}\text { "Áreas" en el Sistema Nacional } \\
\text { de Investigadores (I\&D) }\end{array}$ \\
\hline 1996 & $\begin{array}{l}\text { áreas: ingeniería, ciencias naturales, médicas y } \\
\text { agropecuarias; ciencias sociales; humanidades. }\end{array}$ & $\begin{array}{l}\text { áreas: ciencias físico- } \\
\text { matemáticas; ciencias biológicas, } \\
\text { biomédicas y químicas; ciencias } \\
\text { sociales y humanidades; } \\
\text { ingeniería y tecnología. }\end{array}$ \\
\hline 1999 & $\begin{array}{l}5 \text { áreas: ingeniería y tecnología; ciencias } \\
\text { agropecuarias; ciencias naturales y exactas; ciencias } \\
\text { sociales y humanidades; ciencias de la salud. }\end{array}$ & $\begin{array}{l}4 \text { áreas: ciencias físico- } \\
\text { matemáticas; ciencias biológicas, } \\
\text { biomédicas y químicas; ciencias }\end{array}$ \\
\hline
\end{tabular}




\section{Las nuevas narrativas, en el entorno social Universidad de La Laguna, diciembre de 2019}

\begin{tabular}{|c|c|c|}
\hline & & $\begin{array}{l}\text { sociales y humanidades; } \\
\text { ingeniería y tecnología. }\end{array}$ \\
\hline $2001-2002$ & $\begin{array}{l}6 \text { áreas: ciencias agropecuarias, exactas y naturales; } \\
\text { ciencias de la salud; ingeniería y tecnología; sociales y } \\
\text { administrativas; educación y humanidades. }\end{array}$ & $\begin{array}{l}7 \text { áreas: físico-matemáticas y } \\
\text { ciencias de la tierra; biología y } \\
\text { química; medicina y ciencias de la } \\
\text { salud; humanidades y ciencias de } \\
\text { la conducta; ciencias sociales; } \\
\text { biotecnología y ciencias } \\
\text { agropecuarias; ingeniería. }\end{array}$ \\
\hline 2010 & $\begin{array}{l}6 \text { áreas: ciencias agropecuarias; ciencias exactas y } \\
\text { naturales; ciencias de la salud; ingeniería y tecnología; } \\
\text { ciencias sociales y administrativas; educación y } \\
\text { humanidades. }\end{array}$ & $\begin{array}{l}7 \text { áreas: ciencias físico- } \\
\text { matemáticas y de la tierra; } \\
\text { biología y química; medicina y } \\
\text { ciencias de la salud; humanidades } \\
\text { y ciencias de la conducta; ciencias } \\
\text { sociales; biotecnología y ciencias } \\
\text { agropecuarias; ingeniería. }\end{array}$ \\
\hline 2016 & $\begin{array}{l}6 \text { áreas: ciencias agropecuarias; ciencias naturales y } \\
\text { exactas; ciencias de la salud; ingeniería y tecnología; } \\
\text { ciencias sociales; humanidades. }\end{array}$ & $\begin{array}{l}7 \text { áreas: ciencias físico- } \\
\text { matemáticas y de la tierra; } \\
\text { biología y química; medicina y } \\
\text { ciencias de la salud; humanidades } \\
\text { y ciencias de la conducta; ciencias } \\
\text { sociales; biotecnología y ciencias } \\
\text { agropecuarias; ingeniería. }\end{array}$ \\
\hline
\end{tabular}

Fuente: elaboración propia con base en informes del Conacyt, 1996-2016.

Para ser sinceros, eran de esperarse mayores transformaciones estructurales en el ordenamiento epistémico dadas las propuestas e iniciativas respecto de la supradisciplina, tanto en investigación como en educación (Cunningham, 1999). Pero esa estructura que se observa desde 2001 se explica porque los criterios de la OCDE se imponen como los óptimos para para medir los recursos humanos dedicados a ciencia y tecnología.

El documento de la OCDE, conocido como "Manual de Canberra", fue emitido en 1995 y el Conacyt se comprometió a observarlo, junto con el "Manual de Frascati", para el diseño y evaluación de las políticas públicas en su materia. En tanto instrumento para "clasificar a los individuos de acuerdo con su bagaje educativo", el 


\section{Las nuevas narrativas, en el entorno social Universidad de La Laguna, diciembre de 2019}

Manual de Canberra especifica los "campos" convenientes a la economía basada en el conocimiento, constituyéndolos en estándares internacionales a los que México decidió apegarse (OCDE, 1995: 84).

Es en ese sentido, y con este argumento, que al observar la indización del conocimiento contemporáneo incluido en el Manual no parecen reflejarse los debates que se refieren a la inter y la transdisciplina, sobre todo en términos de procurar salidas a la lógica disciplinar por las razones que formuló Nicolescu: Si a la interdisciplina "le concierne la transferencia de métodos de una disciplina a otra... la transdisciplina se ocupa de lo que está entre las disciplinas, a través de las disciplinas y más allá de las disciplinas" (Nicolescu, 2008: 2).

Para dejar las cosas claras, señalemos que esta es la estandarización internacional en torno a los "campos de ciencia y tecnología" tal como las nombra el Manual: 1. ciencias naturales; 2. ingeniería y tecnología; 3. ciencias médicas; 4. agricultura; 5 . ciencias sociales; 6 . humanidades.

Obviamente cada rubro incluye varias disciplinas con denominaciones tradicionales (con énfasis monodisciplinar). Tomemos por caso a las Ciencias Sociales. En ellas se incluyen: "psicología, economía, ciencias educativas, otras ciencias sociales (antropología y etnología, demografía, geografía, planeación urbana y rural, administración, derecho... ciencias sociales diversas y actividades de ciencia y tecnología interdisciplinarias, metodológicas e históricas relacionadas con materias de este grupo)" (OCDE: 1995: 90, cursivas nuestras).

La inclusión de la interdisciplina en el concepto de "otras ciencias sociales" no deja lugar a dudas de la escasa relevancia que se le reconoce, y no se condice con la vasta producción teórica que ha habido al respecto. Mucho menos si tomamos en cuenta que la Unesco ha sido un organismo internacional muy destacado en la promoción de la transdisciplina como política educativa y de investigación (Nicolescu, Ibid). Visto el estado de la discusión académica, cabría esperar algo de lo que Foucault caracterizaba para las épocas de cambio de discursividad: 


\section{Las nuevas narrativas, en el entorno social Universidad de La Laguna, diciembre de 2019}

"La transformación de una práctica discursiva está ligada a todo un conjunto, a menudo muy complejo, de modificaciones que pueden producirse ya fuera de ella (en las formas de producción, en las relaciones sociales, en las instituciones políticas), ya en ella (en las técnicas de determinación de los objetos, en el afinamiento y el ajuste de los conceptos, en la acumulación de la información), ya al lado de ella (en otras prácticas discursivas). Y está ligada a las mismas bajo la forma, no de un simple resultado, sino de un efecto que detenta a la vez su propia autonomía, y un conjunto de funciones precisas en relación con lo que la determina" (Foucault, 2016: 109).

Tal vez en eso resida la gran cuestión. En la ausencia de remisiones a nuevas formas de producción y determinación de los objetos de acuerdo con otras aspiraciones, menos apegadas a la tradición de los "dominios" de la gestión del conocimiento (Ávila y Andrade: 71). Da la sensación de que Sísifo se dirige a la cima sin saber qué hará después con su roca, a diferencia de la interpretación de Camus. En otras palabras: la existencia de nuevos materiales y desarrollos tecnológicos permanece encapsulada en las determinaciones (los "para qué") de la modernidad científica, con todo y los riesgos que han sido profusamente señalados por gente como Beck, Giddens, Lash y Leff.

La innovación de protocolos, productos y rendimientos tecnofinancieros no puede seguir siendo el leit motiv de la acción científica. La ciencia debe salir de sí, en un extrañamiento radicalmente nuevo, si quiere conservar la legitimidad que ha alcanzado como esfera institucional. Las aporías están pendientes de los nuevos tiempos, en un vuelco paradójico hacia el "todo se vale" mientras sea rentable, arbitrio del que un día la ciencia soñó escapar. Y ese sueño, como en Goya, ha producido más de un monstruo.

"La disciplina es complementaria de los nuevos abordajes", han dicho Nicolescu y sus colaboradores con su compromiso declarado hacia la huidiza unidad de la ciencia (si bien principalmente a título de un ethos compartido e inscrito en la noción de complejidad). Ése es sin duda uno de los puntos de máxima dificultad para su 


\section{Las nuevas narrativas, en el entorno social Universidad de La Laguna, diciembre de 2019}

proyecto: alinear a la ciencia con la filosofía y no en su contra, lo que no han hecho posible ni siquiera con el concurso del mito, porque el mito es el "saber originario" y depende enteramente "de los dioses y la naturaleza", lo que lo hace difícilmente administrable ab initio.

También en 1995, como el Manual de la OCDE, la Comisión Europea emitió una iniciativa de los Países Bajos para estimular la oferta de "oportunidades a nuevos esfuerzos interdisciplinares, lo que significaba abrir el sistema y hacerlo flexible" (Cunningham: 121). Teniendo como foco los procesos de investigación, sugiere que tanto la monodisciplinariedad como la interdisciplinariedad pueden atender eficazmente problemas de esa índole, ya sean definidos de manera endógena o exógena al dominio de la ciencia, y que de hecho ambas pueden combinarse con éxito en función de esas definiciones.

Es una confirmación de que, en la etapa actual, las instancias de gestión de la llamada economía basada en el conocimiento no han resuelto abandonar la monodisciplina, ni optar por formas alternativas de organización de los saberes socialmente constituidos, sino por una combinatoria de los conocimientos especializados, según la definición instituida de los problemas epistémicos y sociales, y sus modos de regulación y evaluación en términos binarios.

Figura 1. Enriquecer el conocimiento, Países Bajos.

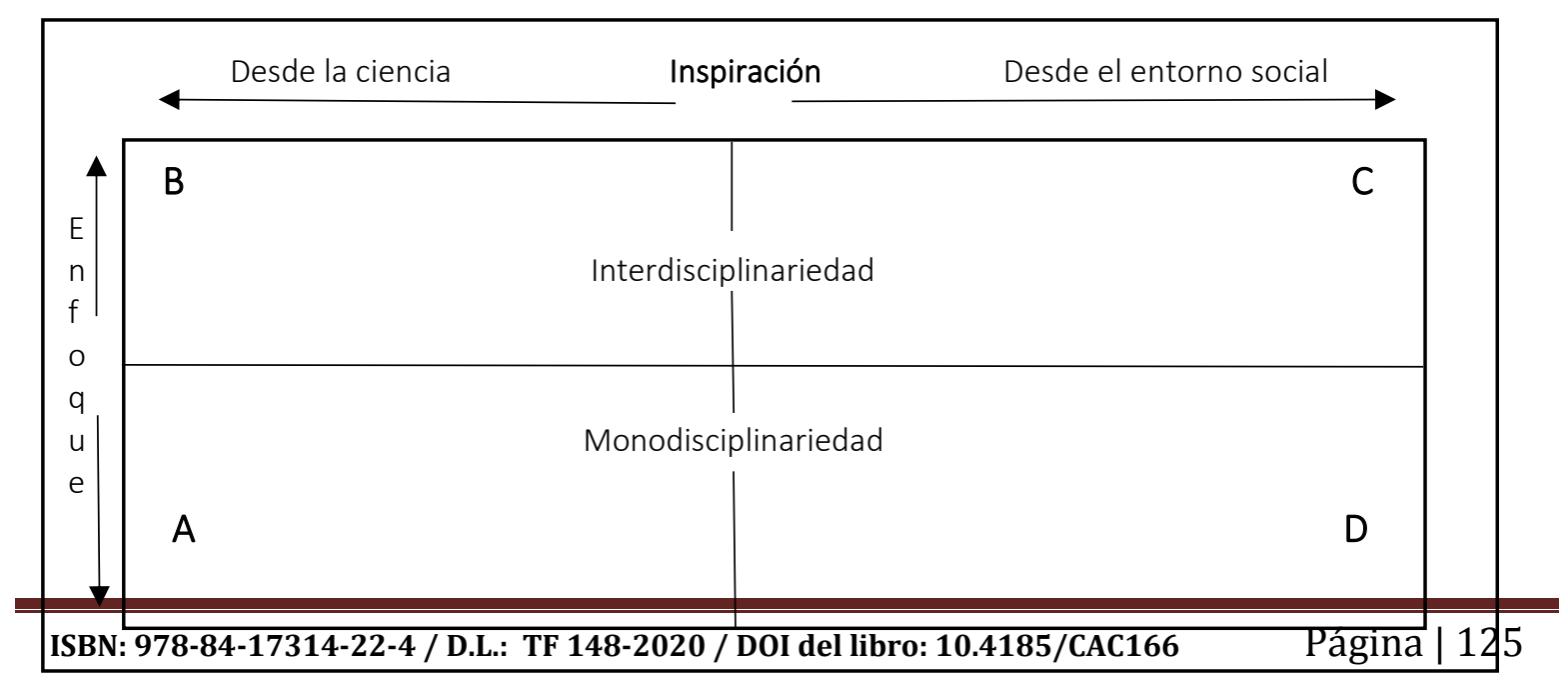

Libro colectivo en línea: http://www.revistalatinacs.org/19SLCS/libro-colectivo-2019.html 


\section{Las nuevas narrativas, en el entorno social Universidad de La Laguna, diciembre de 2019}

Fuente: Cunningham, R., Interdisciplinarity and the organization of knowledge in Europe.

No deja de ser interesante, ni está de más repetirlo, que una de las mayores aportaciones de los practicantes de la interdisciplina, así como de los impulsores de la perspectiva transdisciplinar, consiste en su negativa a deponer la crítica epistemológica, lo que favorece la posibilidad de "una cooperación inestable y unos acercamientos metodológicos que no se conformen con exigencias estrictamente pragmáticas. Esa toma de posición implica una ética común (un éthos) como dominio de la acción humana que condiciona también a la episteme clásica tanto como al saber productivo de base tecnológica" (Ávila y Andrade: 76).

Una actitud de esa clase conduce directamente a interrogar las relaciones que establecen entre sí los tres "dominios" que aquí nos ocupan. Sin embargo, nuestra primera precaución es evitar reificarlos. Ni la comunicación, ni la cultura se deducen del estatuto organizacional, cualquiera que éste sea, debido esencialmente a que un estatuto tal tiene que ser eminentemente determinado, y este es un hecho que suele pasarse por alto. Por esa razón, al investigar acerca del constructo social llamado "organización", hemos optado por hacerlo de manera directa, preguntando por su sentido inmediato, fenoménico, atentos a la formulación de la cuestión y no tanto a la respuesta preconcebida que nos adviene de la cultura académica y profesional.

\section{Dos hipótesis acerca de la organización.}

¿Qué implicaciones tiene esta situación sobre nuestra materia organizacional y cómo el problema de la posdisciplina hace patente la necesidad de renovación de la comunicación en organizaciones formales? Esperamos que se aclare un poco mostrando el origen de este problema epistémico. 


\section{Las nuevas narrativas, en el entorno social Universidad de La Laguna, diciembre de 2019}

A primera vista, interrogar directamente parece no ser un procedimiento fértil. Preguntas del tipo ¿qué es la organización? llevan necesariamente a la cuestión de la identidad y, junto con ella, a la de esencia. Nos ponen de lleno en el terreno de la ontología, y nosotros lo que deseamos es evitar incurrir, ab initio, en esa clase de especificaciones.

No obstante, creemos que ese derrotero no ha tenido resultados porque sus preguntas no han sido las adecuadas o no suficientemente radicales. Por ejemplo, preguntar "¿qué es la organización?" presupone injustificadamente la existencia empírica de una cosa tal y con "tales" características que, sin embargo, no ha sido determinado discreta y deliberadamente. $Y$ pues consideramos que las preguntas deben radicalizarse, queremos indagar acerca del fáctum, del hecho en cuanto que sujeto de experiencia y, en términos kantianos, de juicio reflexionante, que pregunta por lo empíricamente dado y construye paulatinamente la concepción de sus objetos al encarar sus propiedades que desbordan las fronteras mecánicas de la naturaleza en favor de formulaciones teleológicas.

Postulemos desde lo más básico: a) las organizaciones que interesan a la Teoría de las organizaciones, así como a la Cultura y Comunicación organizacionales, son constructos, tanto empíricos como conceptuales, lo cual implica que han sido dotados de sentido desde fuera de sí mismas. Este primer aspecto aparta de nuestro punto de vista la concepción reificadora de Luhmann en cuanto que las organizaciones son sistemas autopoiéticos, cosa que discutiremos más adelante. b) Para cobrar sentido de realidad humana, es decir, en este caso, para incorporarse de lleno al mundo de la intencionalidad, es absolutamente preciso efectuar el deslinde teórico entre la entidad compuesta y la unitaria, lo que ya supone un debate abierto acerca del problema de la identidad organizacional y, desde luego, de las posibilidades fácticas de construir una armonía identitaria.

c) Las profundas divergencias acerca del objeto organización reflejan sesgos ideológicos y perceptuales, así como adhesiones a ciertos esquemas de 


\section{Las nuevas narrativas, en el entorno social Universidad de La Laguna, diciembre de 2019}

construcción de la realidad y concordancia con modelos políticos y económicos específicos (Perrow, 1984: 29-314). Las organizaciones tienen adjudicados fines, eso es incontrovertible. Es propio de la acción, individual y colectiva, la persecución de objetivos que se desean alcanzar (Weber, 2002: 5-45). Nos parece aquí que el principal problema radica en el horizonte histórico seleccionado para la determinación de los fines organizacionales (Etzioni, 1989). Sin esos cortes históricos, más aún, sin explicitar las razones por las cuales se eligen, toda determinación de fines resulta arbitraria e injustificada. De esa manera, situar en la modernidad el grado cero de las organizaciones formales implica hacerles extensivos las afanes y propósitos de la burguesía, naciente o consolidada, con la nueva división de clases que viene aparejada con esa emergencia histórica, lo cual no es cosa menor para efectos del análisis.

Bien, dicho esto debemos precisar nuestras dos hipótesis, mutuamente excluyentes:

1) Las organizaciones existen como espacios psicosociales de acción;

2) Las organizaciones existen como sistema que, en realidad, no puede incluir en sí las exigencias de la hipótesis anterior y, en consecuencia, no pueden ser tratadas como entidades por carecer de unidad.

Aun procediendo a añadir el tercero excluido, tal como sugieren los defensores del pensamiento transdisciplinar: las organizaciones, en tanto constructos, existen como realidad simbólica y material, y a pesar de que no es posible armonizar ambas dimensiones (pues contrario a lo que pensaban, digamos, Roethlisberger y Dickson, no son de suyo armonizables en un Todo absoluto) asumen realidad en la experiencia de los sujetos con los que interactúan y reconocen en ellas ese mismo carácter. De todos modos, plantearlo como propósito último de la gestión o la investigación es incorporarlo ya, anticipadamente, en una de las corrientes hegemónicas que otorgarían validez a los resultados propuestos y a los realmente alcanzados. 


\section{Las nuevas narrativas, en el entorno social Universidad de La Laguna, diciembre de 2019}

\section{Organización como objeto de la acción y organizaciones como sujeto.}

Lo que vamos a intentar es solo una propuesta de interpretación y un primer atisbo a las posibilidades de desontologizar a las organizaciones formales y, con ellas, a la comunicación organizacional.

A lo largo de nuestro trayecto en el dominio de ésta última, hemos enfatizado la necesidad de su renovación epistemológica y práctica. Por desgracia, la experiencia demuestra que la mayoría de los cambios han sido capilares y lexicales, sin un genuino interés de renovación. Es en este sentido que recordamos aquí la propuesta de Habermas acerca de los intereses epistemológicos (2009: 159-181). Como es sabido, Habermas rechaza que el conocimiento se aborde o se produzca con ánimo desinteresado y estrictamente contemplativo, tal como Kant, y mucho antes Aristóteles, afirmaron partiendo de la interpretación de theoria como contemplación, y deponiendo a su vez la de "investigación", que también le corresponde desde sus orígenes.

En la producción de conocimiento pueden distinguirse tres tipos de interés, en un esquema weberiano de tipos ideales: primero, un interés del científico en la selección metodológica y la producción epistémica de contenidos y métodos técnicos. Los resultados de la ciencia obedecen a la reproducción, ampliada o simple, del estado de la técnica, pero ante todo obedece a los mismos imperativos instrumentales de la reducción tecnocientífica. Un reduccionismo tal amplía las brechas en la distribución de la riqueza y abona a una mayor desigualdad educativa, cognoscitiva y social. Este es el principal interés con el que han sido abordadas la teoría de las organizaciones y la comunicación organizacional, constituyéndose, en los hechos, como hegemón que dificulta enormemente otro tipo de abordajes. Pero no los hace imposibles.

El segundo interés epistémico es el emancipatorio. Su sentido estricto es el formulado por Kant en la pregunta por la Ilustración y la Metafísica de las 


\section{Las nuevas narrativas, en el entorno social Universidad de La Laguna, diciembre de 2019}

costumbres. En ellos Kant defiende lo que ya antes habían propugnado Etienne de La Boétie y Baruch Spinoza: la autonomía de pensamiento y opinión, condición indispensable para la legitimidad de un Estado y una sociedad libre. Emancipación, entonces, queda aclarado, significa para nosotros la autonomía de los individuos para poder juzgar y decidir; es decir, para ejercer la crítica, pues no olvidemos que kritiké es, antes que todo y al margen de su significado médico, tomar una decisión, juzgar, ejercer la facultad judicativa de la razón y la comunicación con nuestros semejantes, a lo que Kant llama humanitas práctica, que "es libre y consiste, por tanto, en compartir (communio sentiendi liberalis) y se fundamenta en la razón práctica" (Kant, 2008: 328).

El tercer interés epistémico, al que el propio Habermas sitúa en segundo lugar pero que, por lo que acabamos de decir, nosotros juzgamos definitorio de los otros dos, es el interés práctico. En él se reconocen las distintas ciencias como históricas o instrumentales; técnicas o teóricas. Esa dimensión histórica implica un retorno a lo que señalábamos antes: es en su horizonte histórico en el cual, y desde el cual, una ciencia adquiere su estatuto de validez y de verdad. La posibilidad de interpretar el locus de la ciencia, con sus métodos técnicos y hermenéuticos en igualdad de condiciones, hace del interés práctico uno de inmenso valor para el estudio y ejercicio de la comunicación organizacional. Así lo muestra el cuadro siguiente, que distingue entre la acción racional con arreglo a fines, presuntamente propia de las organizaciones formales, y otras formas de interacción simbólicamente mediadas: 


\section{Las nuevas narrativas, en el entorno social Universidad de La Laguna, diciembre de 2019}

Los sistemas sociales de acuerdo con sus tipos de acción.

\begin{tabular}{|c|c|c|}
\hline & $\begin{array}{l}\text { Marco institucional: } \\
\text { interacción } \\
\text { simbólicamente } \\
\text { mediada. }\end{array}$ & $\begin{array}{l}\text { Sistemas de acción } \\
\text { racional con arreglo a } \\
\text { fines (instrumental y } \\
\text { estratégica). }\end{array}$ \\
\hline $\begin{array}{l}\text { Reglas orientadoras de la } \\
\text { acción. }\end{array}$ & Normas sociales. & Reglas técnicas. \\
\hline Niveles de definición. & $\begin{array}{l}\text { Lenguaje ordinario } \\
\text { intersubjetivamente } \\
\text { compartido. }\end{array}$ & $\begin{array}{l}\text { Lenguaje libre de } \\
\text { contexto. }\end{array}$ \\
\hline Tipos de definición. & $\begin{array}{l}\text { Expectativas recíprocas } \\
\text { de comportamiento. }\end{array}$ & $\begin{array}{l}\text { Pronósticos } \\
\text { condicionados: } \\
\text { imperativos } \\
\text { condicionados. }\end{array}$ \\
\hline $\begin{array}{l}\text { Mecanismos de } \\
\text { adquisición. }\end{array}$ & Internalización de roles. & $\begin{array}{l}\text { Aprendizaje de } \\
\text { habilidades y } \\
\text { cualificaciones. }\end{array}$ \\
\hline $\begin{array}{l}\text { Función del tipo de } \\
\text { acción. }\end{array}$ & $\begin{array}{l}\text { Mantenimiento de } \\
\text { instituciones } \\
\text { (conformidad con las } \\
\text { normas por medio del } \\
\text { reforzamiento recíproco). }\end{array}$ & $\begin{array}{l}\text { Solución de problemas } \\
\text { (consecución de fines } \\
\text { definida en relaciones fin- } \\
\text { medio). }\end{array}$ \\
\hline $\begin{array}{l}\text { Sanciones cuando se } \\
\text { viola una regla. }\end{array}$ & $\begin{array}{l}\text { Castigo basado en } \\
\text { sanciones } \\
\text { convencionales (fracaso } \\
\text { frente a la autoridad). }\end{array}$ & $\begin{array}{l}\text { Ineficacia: fracaso ante la } \\
\text { realidad. }\end{array}$ \\
\hline “Racionalización" & $\begin{array}{l}\text { Emancipación, } \\
\text { individuación; extensión } \\
\text { de la comunicación libre } \\
\text { de dominio. }\end{array}$ & $\begin{array}{l}\text { Aumento de las fuerzas } \\
\text { productivas; extensión del } \\
\text { poder de disposición } \\
\text { técnica. }\end{array}$ \\
\hline
\end{tabular}

Se aprecia claramente que el momento de la "racionalización" obedece a la intensificación de las fuerzas productivas y su incremento continuo de la eficacia. Desde el punto de vista fenomenológico, un proceso de esa clase no puede obtener su legitimidad sino de la dimensión preteórica, dado que al ejercer la crítica sometiéndolo a uno reflexivo (de segundo o tercer orden, es decir, uno en el que al observar el observador se observa a sí mismo, y a su vez se sabe observado por otro observador) da muestras de un mundo de la vida colonizado por las fuerzas sistémicas que se oponen a una comunicación "libre de dominio". 


\section{Las nuevas narrativas, en el entorno social Universidad de La Laguna, diciembre de 2019}

Ahora bien, Supongamos que en la literatura del área organizacional se oponen dos posturas primarias: agnosticismo y naturalismo. Una postulando la imposibilidad del conocimiento verdadero derivado de la experiencia y teorización organizacional; el otro evadiendo lecturas ontológicas y delimitando el valor de verdad al éxito de las acciones. Por ejemplo, Rolando García dice en "Interdisciplinariedad y sistemas complejos" (2011: 99): "Los sistemas complejos se comportan como 'totalidades' compuestas de subsistemas", de modo tal que adopta como punto de partida una serie de presupuestos que no han sido demostrados ni de manera lógica ni de manera empírica. Esta forma de proceder es parte de la ciencia ingenua, que hace suyos, a modo de principios, aforismos de una auctoritas disciplinar que a lo largo del tiempo se va decantando hasta sedimentarse como verdad corriente.

Un punto de vista diferente lo proporciona Luis Montaño:

"El objeto de la teoría de la organización es diverso, dinámico, complejo y difuso. Es diverso por la amplia gama de organizaciones que contempla: empresas, universidades, estatales, Estado, etcétera. Pero lo es también por sus propias características en términos de, por ejemplo, avance tecnológico, número de empleados, régimen de propiedad, función social... La organización podrá ser considerada así como un conjunto diverso de lógicas tanto de comportamiento como de reapropiación de lo real. El objeto es dinámico por el conjunto de elementos que lo predeterminan. Los grandes cambios en términos de modelo económico, político, tecnológico, social, e incluso cultural, están constantemente presentes y se expresan como presión al cambio, del cual, hay que agregar inmediatamente, las organizaciones son también productoras... Es complejo no sólo por el número de variables que intervienen, sino por la diversidad de dimensiones que alberga en su unidad: técnica, política, económica, simbólica, lingüística, etcétera. Finalmente, es también difuso por la dificultad de equiparar fronteras físicas y legales con 


\section{Las nuevas narrativas, en el entorno social Universidad de La Laguna, diciembre de 2019}

sociales. El traslado metafórico reformula los espacios sociales, permitiendo el traslado epidemiológico -y por ende, metafórico- de un campo a otro" (Montaño, 2000: 79, cursivas nuestras).

En el primer caso, García parte del supuesto, únicamente postulado, de que las organizaciones se comportan como sistemas que, a su vez, se componen de subsistemas. El órganon se establece como lógica de los fines y la autarquía biofísica. Este es el canon de la instrumentalidad. La distinción que suele hacerse entre organizaciones-máquina y organizaciones-organismo se condensa y resume en esta perspectiva, con la diferencia notable de que en el primer caso la disposición de las funciones se ejerce desde un sujeto situado en un plano superior, y en el segundo son los mecanismos homeostáticos los que autorregulan a la totalidad biológica. En ambos casos, sin embargo, la elección es clara en cuanto a considerar a la organización como unidad compuesta de partes, ya físicos, ya orgánicos.

Montaño, por su parte, lleva a cabo un giro fundamental, si bien nos parece todavía insuficientemente sustentada. El esfuerzo que lleva a cabo se concentra en lo que Aristóteles llama accidentes: la predicación del ente que se pretende conceptualizar, esto es, definir. Creemos que la ruta para poder asir lo propio de las organizaciones (entendiendo además que lo "propio", de acuerdo también con Aristóteles, no define a la entidad "organización") no pasa por describir ni las características físicas ni legales ni sociales, e incluso tampoco simbólicas.

Si hemos de ser congruentes, debemos afirmar que la organización, por el solo hecho de serlo, no admite unidad, de ninguna clase. $Y$ eso debido a un hecho notable que presenta Benveniste (1973: 213):

"No hubo a mediados del siglo XVIII más que un número reducidísimo de creaciones de este orden... apenas se encuentran [en lengua francesa] fertilisation, thésaurisation, temporisation, organisation (creada ésta 


\section{Las nuevas narrativas, en el entorno social Universidad de La Laguna, diciembre de 2019}

anteriormente, pero que no cobró vida hasta entonces) y, en fin, nuestra civilisation... En tan menguado contingente, incluso, la mayor parte de las palabras conservan el sentido exclusivo de 'acto' (así fertilisation). Para pasar a la noción de estado, a la que llega en seguida civilisation, solo puede citarse organisation, en la 'organisation de los vegetales', y luego 'de las organisationes caritativas".

Según indica Benveniste, "en Inglaterra las condiciones son curiosamente parecidas" (Ibid: 214), de modo que debemos remitirnos a un antes de la modernidad si lo que deseamos es comprender no los accidentes del fenómeno "organización", sino la cosa, el algo al que nos referimos cuando decimos que la organización es así o asá, con estas o aquellas características, de esta o aquella forma. Eso será algo que deberemos posponer pues no queremos embarcarnos en un tema que por sí solo abarca un tratado. Lo que sí es cierto, es que tal vez amerita la creación de una organología como estudio y discurso del hecho organizado.

Y aquí proponemos esto como nuestra clave de acceso a la comprensión de las organizaciones: el estudio de la Forma, el eidos, la esencia de la organización que debe ser expuesto proposicionalmente por medio del hórismos, definición que dice (légein) lo que la organización es en cuanto organización.

En este desplazamiento del objeto, es preciso efectuar un descentramiento del sujeto en favor de la interacción como instancia propia del verbo organizar. ¿Qué es un hecho organizado? Más aún, ¿qué es organizar? Cuáles son los presupuestos, habitualmente implícitos, por supuesto, que para afirmar que algo está organizado y algo no lo está. Por supuesto, ¿qué es ese algo?

\section{Comunicación y organización, bases para un compendio de relaciones inestables.}




\section{Las nuevas narrativas, en el entorno social Universidad de La Laguna, diciembre de 2019}

El espíritu de estos trabajos nació de las Lógicas de Hegel y Aristóteles, así como de la kantiana Crítica del Juicio y, quizá sobre todo, de una lectura del Crátilo de Platón. En éste último, Platón ofrece un indicio sumamente valioso para entender el título de Organon a la Lógica de Aristóteles (debemos precisar que es hasta la baja Edad Media que se adopta ese nombre para la Lógica aristotélica: "Organon", instrumento, medio, útil), llamándolo también artilugio, mecanismo, intención de engaño. El organon, pues, se conecta directamente con la texné, la técnica entendida como actividad poiética y no meramente práctica.

La actividad (energeia) transforma la potencia poniéndola en acción y permitiendo la producción (poiesis) de cosas que "se traen al mundo" dado que no existen con anterioridad. El resultado es "algo" distinto al agente, como Aristóteles ha dejado en claro. Bien, es ahí en dónde la Lógica de Hegel interviene: "En la existencia se ha distinguido su determinación como cualidad: en esta, como existente, se halla la diferencia... De este modo, la cualidad en general no se halla separada de la existencia, que es solo un ser determinado y cualitativo" (Hegel, 2013: 146).

Repitámoslo: "un ser determinado y cualitativo". Antes, al inicio de esta obra, Hegel hizo dos afirmaciones radicales sobre las que sustenta su ontología: El ser sin determinaciones es la nada, ni más ni menos que la nada; mientras que la nada es la ausencia de indeterminaciones y, por consiguiente "la indistinción de sí misma".

Determinar al ser, predicarlo del ente, fenomenizarlo y permitir, solo entonces, el trabajo hermenéutico fundamentado en lo fáctico. "Hermenéutica de la facticidad", ha llamado Heidegger a su propia ontología.

Esa es la facticidad en la que existe el humano, el mundo de la vida en que se producen las interacciones y se hace posible toda comunicación. Porque la comunicación no es una elección libre y autárquica: en la comunicación se vive, es condición humana por excelencia. Al inicio de la Política, Aristóteles postuló que el humano es un animal social (zoé politikón), pero lo es debido a que está dotado de palabra (lógos). Si bien a nuestro parecer lo distintivo del humano es trabajo (ergon) 


\section{Las nuevas narrativas, en el entorno social Universidad de La Laguna, diciembre de 2019}

y comunicación, lo que ha dado lugar a la formación de sociedades cada vez mayores a partir de unidades gregarias formadas por homínidos, a pesar de creer esto, nos parece indudable que sin comunicación la humanidad es impensable e indecible.

Lo mismo pasa con las organizaciones. La táxis griega, el poner orden, el dis-poner o diáthesis. La comunicación está lejos de ser un dispositivo que se pueda gestionar al gusto del director en turno. La comunicación es una héxis, un modo de vida. Más aún, sin comunicación la organización no es nada, dicho absoluta y radicalmente. Nos hubiera gustado exponer aquí algunas ideas que tenemos al respecto, pero deberemos dejarlo para un trabajo más extenso y razonado. Y sí, por supuesto, pensando a los sistemas como metáforas de lo real, pero más precisamente de lo compuesto (lo complejo) que deviene problemático cuando se le quiere dar un tratamiento semejante a lo simplex o unitario.

\begin{tabular}{|c|c|c|}
\hline $\begin{array}{l}\text { Categoría } \\
\text { de acción }\end{array}$ & $\begin{array}{l}\text { Relaciones de } \\
i=\text { interacción }\end{array}$ & $\begin{array}{c}\text { Relaciones de } \\
r=\text { representación }\end{array}$ \\
\hline Modalidad & $\begin{array}{l}\text { Directa, procesual, continua, } \\
\text { analógica. }\end{array}$ & $\begin{array}{l}\text { Indirecta, episódica, discontinua, } \\
\text { digital. }\end{array}$ \\
\hline $\begin{array}{l}\text { Atribución de } \\
\text { sentido }\end{array}$ & Intersubjetividad. Instituyente. & $\begin{array}{l}\text { Subjetividad genérica, } \\
\text { objetividad. Instituido. }\end{array}$ \\
\hline Realización & $\begin{array}{l}\text { Semántica, práctica, histórica, } \\
\text { ceremonial. }\end{array}$ & $\begin{array}{l}\text { Representación, mediación. } \\
\text { Saber técnico (expertise). }\end{array}$ \\
\hline Orientación & $\begin{array}{l}\text { Propósito (teleología); } \\
\text { coordinación, cooperación. }\end{array}$ & $\begin{array}{l}\text { Metas u objetivos (estrategia); } \\
\text { cooperación/competencia. }\end{array}$ \\
\hline Regulación & $\begin{array}{l}\text { Regulada por normas, } \\
\text { reconocimiento mutuo } \\
\text { (prestigio/saber). }\end{array}$ & $\begin{array}{l}\text { Regulada por medios, } \\
\text { poder/dinero (estructura, control). }\end{array}$ \\
\hline Integración & $\begin{array}{l}\text { Racional/valoral/afectiva/tra- } \\
\text { dicionalmente motivada. }\end{array}$ & $\begin{array}{l}\text { Racional/tradicionalmente } \\
\text { motivada. }\end{array}$ \\
\hline Identidad & Pertenencia (motivación & Afiliación (motivación \\
\hline
\end{tabular}

ISBN: 978-84-17314-22-4 / D.L.: TF 148-2020 / DOI del libro: 10.4185/CAC166

Página | 136 


\section{Las nuevas narrativas, en el entorno social Universidad de La Laguna, diciembre de 2019}

\begin{tabular}{|c|c|c|}
\hline & intersubjetiva). & externa/genérica). \\
\hline Interés práctico & $\begin{array}{l}\text { Comunidad de intereses } \\
\text { (sociabilidad, incluye el trabajo } \\
\text { como work, homo faber). }\end{array}$ & $\begin{array}{l}\text { Intereses funcionalmente } \\
\text { motivados (prima el trabajo como } \\
\text { job, homo laborans). }\end{array}$ \\
\hline
\end{tabular}

Fuente: elaboración propia.

La acción puede ocurrir, en este marco, ya sea como relaciones mediadas por la representación, o realizadas mediante interacciones. La distinción entre ellas conlleva un tratamiento distinto al binomio comunicación-organización.

Visto desde la representación, la comunicación es congelada como dispositivo susceptible de operar de acuerdo con intenciones planificadoras y operacionales. En cambio, desde las cualidades que ofrece la interacción, la organización, su táxis y su héxis, es del todo contingente a la acción comunicativa y no puede afirmarse de ningún otro modo, ni en términos epistémicos, ni en términos profesionales.

\section{Comentarios finales.}

Valga lo último como gozne que, al tiempo que cierra este trabajo, abre desde sí nuevos trabajos en desarrollo. Lo hemos dicho antes: o se transforma y renueva, o la comunicación organizacional desaparece. En esta disyuntiva, nos parece que la multiplicación de perspectivas resulta muy saludable para consolidar las líneas y tendencias predominantes, fundamentalmente de orden técnico, pero propiciando también la apertura a otros intereses epistémicos. No creemos, sin embargo, que esa consolidación deba traducirse en una sedimentación disciplinar. Ni siquiera en una nueva cuadratura o ampliación del "campo disciplinar". Al contrario. La fortaleza de la comunicación organizacional debe alimentarse de la ruptura de fronteras epistémicas y el abandono de las antiguas pretensiones de encontrar un lugar especial y propio en la organización institucional del saber. 


\section{Las nuevas narrativas, en el entorno social Universidad de La Laguna, diciembre de 2019}

La comunicación debe estar libre de condicionamientos instituidos; las ciencias de la comunicación no han hallado su centro de gravedad y sin embargo multiplican sus objetos, cuestiones e intereses. La comunicación organizacional deberá, de modo equivalente, sustraerse a la influencia organizativa para hacerse cargo, al fin, del problema organizacional.

\section{Bibliografía.}

- Aristóteles (2015a). Tratados de lógica. Gredos: Madrid. (2015b). Metafísica. Gredos: Madrid.

- Ávila, R. (2014). "Comunicación y cultura: problemas emergentes en organizaciones de trabajo". En Meneses, M. et al. (coord.), La investigación de la comunicación ante el nuevo marco regulatorio de las telecomunicaciones. UASLP: México, pp. $1103-1117$.

y P. Schiaffini (2013). "Dimensiones culturales del sentido en la investigación de la comunicación organizacional: apreciaciones generales." En Aguilar, A. et al., (coord.), La producción del conocimiento en las ciencias de la comunicación y su incidencia social. (2013), UAC: México, pp.171-199.

y A. Andrade (2019). "Organización, cultura y comunicación: un acercamiento a las opciones supradisciplinares". Organicom, Revista brasileira de comunicacao organizacional e relacoes públicas. Año 19, No. 30. 2019. Pp. 65-81.

- Benveniste, É. (1973). Problemas de lingüística general. Siglo XXI: México.

- Conacyt (1996-2016). Informes anuales. Conacyt: México.

- Cunningham, R. (1999). Interdisciplinarity and the organization of knowledge in Europe. European Communities: Belgium.

- Etzioni, A. (1989). Organizaciones modernas. UTEHA: México.

- Foucault, M. (2016). Arqueología del saber. Siglo XXI: México. 


\section{Las nuevas narrativas, en el entorno social Universidad de La Laguna, diciembre de 2019}

- García, R. (2011). "Interdisciplinariedad y sistemas complejos". En Revista Latinoamericana de metodología de las ciencias. Vol. 1, No. 1, pp. 65-101.

- Habermas, J. (2009). Ciencia y técnica como ideología. Tecnos: Madrid.

- Kant, I. (2008). Metafísica de las costumbres. Tecnos: Madrid.

- Montaño, L. (2000). "La noción de organización: sentido, polisemia y construcción social". En Iztapalapa. Año 20, No. 48. UAM: México, pp. 63-84.

- Nicolescu, B. (2008) Transdisciplinarity: theory and practice. Hampton Press: USA. - OCDE (1995). Manual on the measurement of human resources devoted to C\&T "Manual de Canberra". Http://www.conicyt.cl/wp-content/uploads/2014/07/ManualdeCanberra.pdf

- Perrow, Ch. (1984). "'La historia del zoológico' o 'la vida en el arenal organizativo"'. En Salaman, G. y K. Thompson, Control e ideología en las organizaciones. FCE: México, pp. 293-314.

- Platón (2015). “Crátilo". Diálogos II. Gredos: Madrid, pp. 357-455.

- Weber, M. (2002). Economía y sociedad. FCE: México. 